Chirurg 2019 $\cdot 90: 1026$

https://doi.org/10.1007/s00104-019-01064-y

Online publiziert: 28. Oktober 2019

(c) Springer Medizin Verlag GmbH, ein Teil von Springer Nature 2019

\section{Originalpublikation}

Kurokawa Y, Takeuchi H, Doki Y, Kitagawa Y et al (2019) Mapping of lymph node metastasis from esophagogastric junction tumors. Ann Surg, https://doi.org/10.1097/SLA. 0000000000003499 (Epub ahead)

Hintergrund und Fragestellung. Nach der Klassifikation von Siewert werden die Karzinome des gastroösophagealen Übergangs in drei Typen (AEG Typ I-III) eingeteilt. In internationalen Umfragen wird das Adenokarzinom (AC) des distalen Ösophagus (Typ I) über einen transthorakalen Zugang ösophagektomiert und das subkardiale Magenkarzinom (Typ III) transhiatal erweitert gastrektomiert. Für das Kardiakarzinom (Typ II) sind die Empfehlungen für das operative Vorgehen nicht einheitlich, da das nodale Metastasierungsmuster und damit das Ausmaß der Lymphadenektomie (LAD) nicht eindeutig geklärt sind. Zur Frage der Lymphknotenmetastasierung des Kardikarzinoms wurden jetzt die Ergebnisse einer japanischen prospektiven Beobachtungsstudie vorgestellt.

Methoden. Zwischen 2014 und 2017 wurde bei insgesamt 358 Patienten mit Adeno- und Plattenepithelkarzinom (Tumorzentrum innerhalb $2 \mathrm{~cm}$ der anatomischen Kardia) eine standardisierte, systematische LAD durchgeführt. Der Zugang des chirurgischen Eingriffs wurde in Abhängigkeit von der Tumorausdehnung in den distalen Ösophagus gewählt $(<3 \mathrm{~cm}$ transhiatale Resektion mit limitierter LAD des Mediastinums, $>3 \mathrm{~cm}$ transthorakale Resektion mit ausgedehnter LAD). Die abdominelle LAD war für alle Eingriffe gleich. Primärer

W. Schröder · C. J. Bruns

Klinik für Allgemein-, Viszeral- und Tumorchirurgie, Universitätsklinik Köln, Köln, Deutschland

\title{
Lymphknotenmetastasierung beim Kardiakarzinom
}

Endpunkt war die Rate der LK-Metastasierung (LK-MR) bezogen auf jede einzelne Lymphknotengruppe, die dann für eine von drei Empfehlungskategorien zur LK-Dissketion herangezogen wurde (Kategorie 1 [>10\% LK-MR]: „strongly recommended for dissection“; Kategorie 2 [5-10\% LK-MR]: „weekly recommended“; Kategorie 3 [ $<5 \%$ LK$\mathrm{MR}]$ : „not recommended“).

Ergebnisse. Kategorie-1-LK waren lokalisiert an der kleinen Kurvatur (Station 1 und 3) und am Magenfundus (Station 2), weiterhin an der A. gastrica sinistra, am Tr. coeliacus und an der A. lienalis (Station 7, 9 und 11p). Lymphknoten an der A. hepatica (Station 8) und am gastroösophagealer Übergang wurden als Kategorie-2-LKklassifiziert; LK des unteren Mediastinums (Station 110) wurden bei ösophagealem Tumor von $>2 \mathrm{~cm}$ der Kategorie 1 zugeordnet. Trotz einer ösophagealen Tumorlänge von $>3 \mathrm{~cm}$ ( $98 \mathrm{~Pa}$ tienten) konnte bei keiner LK-Gruppen des mittleren und oberen Mediastinums eine Kategorie-1-Empfehlung ausgesprochen werden. Das Metastasierungsmuster wurde nicht durch die neoadjuvante Therapie beeinflusst.

Fazit. Die systematische prospektive Untersuchung beweist eindrücklich die bidirektionale nodale Metastasierung des Kardiakarzinoms, auch wenn aufgrund dieser morphologischen Studie der abdominellen LAD eine größere Bedeutung zukommt. Die LK-Metastasen sind direkt am Tumor des gastroösophagealen Übergangs zu finden und setzen sich an der kleinen Kurvatur zum Tr. coeliacus fort. Die LK-Metastasierung scheint von der Tumorlänge in den distalen Ösophagus abzuhängen, sodass möglicherweise anhand dieser Variable über das Ausmaß der mediastinalen LAD und damit den chirurgischen Zugang zu entscheiden ist.

\section{Korrespondenzadresse}

Prof. Dr. W. Schröder, FACS, FEBS

Klinik für Allgemein-, Viszeral- und

Tumorchirurgie, Universitätsklinik Köln

Kerpener Str. 62, 50937 Köln, Deutschland

wolfgang.schroeder@uni-koeln.de

Interessenkonflikt. W. Schröder und C.J. Bruns geben an, dass kein Interessenkonflikt besteht. 